PROCEEDINGS OF THE

AMERICAN MATHEMATICAL SOCIETY

Volume 140, Number 1, January 2012, Pages 93-98

S 0002-9939(2011)10921-3

Article electronically published on May 16, 2011

\title{
ALGEBRAS WITH RADICAL SQUARE ZERO ARE EITHER SELF-INJECTIVE OR CM-FREE
}

\author{
XIAO-WU CHEN
}

(Communicated by Birge Huisgen-Zimmermann)

\begin{abstract}
An artin algebra is called CM-free provided that all its finitely generated Gorenstein projective modules are projective. We show that a connected artin algebra with radical square zero is either self-injective or CM-free. As a consequence, we prove that a connected artin algebra with radical square zero is Gorenstein if and only if its valued quiver is either an oriented cycle with the trivial valuation or does not contain oriented cycles.
\end{abstract}

\section{INTRODUCTION AND RESULTS}

Throughout $A$ is an artin algebra over a commutative artinian ring $R$. Denote by $A$-mod the category of finitely generated left $A$-modules and by $A$-proj the full subcategory formed by projective modules. Recall that a complex $P^{\bullet}$ of projective $A$-modules is totally acyclic ([3]) if it is acyclic and that for each projective $A$ module $Q$ the Hom complex $\operatorname{Hom}_{A}\left(P^{\bullet}, Q\right)$ is acyclic. An $A$-module $M$ is called a (finitely generated) Gorenstein projective module ([12]) provided that there is a totally acyclic complex $P^{\bullet}$ such that the zeroth cocycle $Z^{0}\left(P^{\bullet}\right)$ is isomorphic to $M$. In this case, the complex $P^{\bullet}$ is called a complete resolution of $M$. In the literature, Gorenstein projective modules are also called modules of G-dimension zero (1), (maximal) Cohen-Macaulay modules (6, 14, 4]) or totally reflexive modules ([3]).

We denote by $A$-Gproj the full subcategory of $A$-mod formed by Gorenstein projective modules. Observe that $A$-proj $\subseteq A$-Gproj. Recall that an algebra $A$ is self-injective if and only if $A$-Gproj $=A$-mod, that is, all modules are Gorenstein projective. This is one extreme case. We consider another extreme case. An artin algebra $A$ is called $C M$-free provided that $A$-Gproj $=A$-proj, that is, all its finitely generated Gorenstein projective modules are projective; compare [16. Recall that an algebra $A$ of finite global dimension is CM-free; see [4, Remark-Notation 3.7]. However, the converse is not true in general.

Let us remark that the class of CM-free algebras is not well understood; see the remarks after [10, Theorem B]. The following problem might be of interest for future research: for a CM-free algebra, are all its (possibly infinitely generated)

Received by the editors June 10, 2010 and, in revised form, November 9, 2010.

2010 Mathematics Subject Classification. Primary 18G25, 16G10, 16G50.

The author is supported by the Special Foundation of the President of the Chinese Academy of Sciences (No. 1731112304061) and by the National Natural Science Foundation of China (No. 10971206). 
Gorenstein projective modules projective? Here, for the notion of arbitrary Gorenstein projective module, we refer to [12] and [4]. This problem is closely related to a conjecture stated in [7]; also see [5].

Recall that an algebra $A$ is connected if it is not a proper direct product of two algebras. Any algebra is uniquely decomposed as a direct product of connected ones. For an algebra $A$, denote by $\mathbf{r}$ its Jacobson radical. The algebra $A$ is said to be with radical square zero provided that $\mathbf{r}^{2}=0$. Such algebras are studied in 2, Chapter X.2].

The aim of this paper is to show that for a connected algebra with radical square zero the study of its Gorenstein projective modules always belongs to the two extreme cases mentioned above.

Theorem 1.1. Let $A$ be a connected artin algebra with radical square zero. Then $A$ is either self-injective or CM-free.

Let us point out that the local case of Theorem 1.1 follows from a result by Menzin [17, Proposition 4], while the commutative case of Theorem [1.1 is well known; see [19, Proposition 2.4]. We remark that a related consideration is taken in [15, Theorem 3.4].

We draw two immediate consequences of Theorem 1.1. Recall that an algebra $A$ is Gorenstein provided that the regular module $A$ has finite injective dimension on both sides (14). Observe that a self-injective algebra is Gorenstein and that an algebra of finite global dimension is Gorenstein.

Corollary 1.2. Let $A$ be a connected artin algebra with radical square zero. Then $A$ is Gorenstein if and only if it is self-injective or it has finite global dimension.

Proof. It suffices to notice the following fact: a Gorenstein algebra is CM-free if and only if it has finite global dimension. This can be deduced from [4, Proposition 3.10(ii)]. Let us remark that it also follows immediately from a general result, due to Buchweitz and Happel, on the singularity category of a Gorenstein algebra ([6. Theorem 4.4.1] and [14, Theorem 4.6]; also see [9, Proposition 3.8]).

In general, there are Gorenstein algebras which are neither self-injective nor of finite global dimension $([14,8])$. For example, the upper triangular matrix algebra $A=\left(\begin{array}{cc}k[x] /\left(x^{2}\right) & k[x] /\left(x^{2}\right) \\ 0 & k[x] /\left(x^{2}\right)\end{array}\right)$ is such a Gorenstein algebra (see [14] and 8, Remark 3.5]). Here $k$ is a field. Observe that the Jacobson radical $\mathbf{r}$ of $A$ satisfies $\mathbf{r}^{3}=0$ and $\mathbf{r}^{2} \neq 0$.

Recall the notion of the valued quiver of an algebra $A$. Choose a complete set of representatives of pairwise non-isomorphic simple $A$-modules $\left\{S_{1}, S_{2}, \cdots, S_{n}\right\}$. Set $\Delta_{i}=\operatorname{End}_{A}\left(S_{i}\right)$; they are division algebras. Observe that $\operatorname{Ext}_{A}^{1}\left(S_{i}, S_{j}\right)$ has a natural $\Delta_{j}-\Delta_{i}$-bimodule structure. The valued quiver $Q_{A}$ of $A$ is defined as follows: its vertex set is $\left\{S_{1}, S_{2}, \cdots, S_{n}\right\}$ (here we identify each $S_{i}$ with its isoclass); there is an arrow from $S_{i}$ to $S_{j}$ whenever $\operatorname{Ext}_{A}^{1}\left(S_{i}, S_{j}\right) \neq 0$, in which case the arrow is endowed with a valuation $\left(\operatorname{dim}_{\Delta_{j}} \operatorname{Ext}_{A}^{1}\left(S_{i}, S_{j}\right), \operatorname{dim}_{\Delta_{i} \text { op }} \operatorname{Ext}_{A}^{1}\left(S_{i}, S_{j}\right)\right)$; here $\Delta_{i}{ }^{\text {op }}$ denotes the opposite algebra of $\Delta_{i}$. We say that the valuation of $Q_{A}$ is trivial if all the valuations are $(1,1)$. Recall that the algebra $A$ is connected if and only if the underlying graph of $Q_{A}$ is connected. For details, we refer to [2, Chapter III.1] and [11, 3.6].

We have the following consequence of Corollary 1.2. Since the proof requires several standard facts on artin algebras, we postpone it to Section 2. 
Corollary 1.3. Let $A$ be a connected artin algebra with radical square zero. Then $A$ is Gorenstein if and only if $Q_{A}$ either is an oriented cycle with the trivial valuation or does not contain oriented cycles.

\section{Proofs of Theorem 1.1 and Corollary 1.3}

In this section we present the proofs of Theorem 1.1 and Corollary 1.3. We will first make some preparations.

Let $A$ be an artin algebra. Recall that for each $A$-module $M$, its syzygy module $\Omega(M)$ is defined to be the kernel of its projective cover $P \rightarrow M$. Recall that in a short exact sequence $0 \rightarrow M^{\prime} \rightarrow P \stackrel{p}{\rightarrow} M \rightarrow 0$ with $P$ projective, we have $M^{\prime} \simeq \Omega(M) \oplus Q$ for some projective module $Q$; moreover, $Q \simeq 0$ if and only if $p$ is a projective cover. For details, see [2, Chapter IV.3].

We have the following easy observation. Recall that $\mathbf{r}$ denotes the Jacobson radical of $A$.

Lemma 2.1. Let $M$ be a Gorenstein projective A-module without projective direct summands. Assume that $\mathbf{r}^{n}=0$ for some $n \geq 2$. Then $\mathbf{r}^{n-1} M=0$.

Proof. From the definition of a Gorenstein projective module, we infer that there exists a short exact sequence $0 \rightarrow M \rightarrow P \stackrel{p}{\rightarrow} M^{\prime} \rightarrow 0$ with $P$ projective. Since $M$ does not have a projective direct summand, the morphism $p$ is a projective cover. In particular, we have $M \simeq \operatorname{Ker} p \subseteq \mathbf{r} P$. Then $\mathbf{r}^{n-1} M \subseteq \mathbf{r}^{n} P=0$.

Recall that $A$-Gproj denotes the full subcategory of $A$-mod formed by Gorenstein projective modules. Recall that $A$-Gproj $\subseteq A$-mod is closed under direct summands, kernels of epimorphisms and extensions; see [1, (3.11)] and [3, Lemma 2.3]. In particular, for a Gorenstein projective module $M$, its syzygy module $\Omega(M)$ is also Gorenstein projective. Since $A$-Gproj is closed under extensions, it naturally becomes an exact category in the sense of Quillen (18). Moreover, it is a Frobenius category, that is, it has enough (relatively) projective and enough (relatively) injective objects, and the class of projective objects coincides with the class of injective objects. In fact, the class of the projective-injective objects in $A$-Gproj equals $A$-proj. In particular, we have that $\operatorname{Ext}_{A}^{n}(G, P)=0$ for $G \in A$-Gproj, $P \in A$-proj and $n \geq 1$. For details, see [4, Proposition 3.8(i)] and [9, Proposition 3.1(1)].

We consider the stable category $A$-Gproj of $A$-Gproj modulo projective modules. Then the assignment $M \mapsto \Omega(M)$ induces an auto-equivalence $\Omega: A$-Gproj $\rightarrow$ $A$-Gproj; see [13, Chapter I.2]. It is well known that for a Gorenstein projective module $M$, it is indecomposable, viewed as an object in $A$-Gproj if and only if $M \simeq P \oplus M^{\prime}$ for a projective module $P$ and an indecomposable non-projective module $M^{\prime}$.

We will need the following fact.

Lemma 2.2. Let $M$ be a Gorenstein projective A-module which is indecomposable and non-projective. Then $\Omega(M)$ is also an indecomposable non-projective Gorenstein projective A-module.

Proof. We have noticed above that the module $\Omega(M)$ is Gorenstein projective. Since the functor $\Omega: A$-Gproj $\rightarrow A$-Gproj is an equivalence, we infer that $\Omega(M)$ is indecomposable in $A$-Gproj. Hence it suffices to show that $\Omega(M)$ has no projective direct summands. 
Choose a short exact sequence $\xi: 0 \rightarrow \Omega(M) \rightarrow P \stackrel{p}{\rightarrow} M \rightarrow 0$ such that $p$ is a projective cover. We assume that $\Omega(M)=P^{\prime} \oplus M^{\prime}$ with $P^{\prime} \neq 0$ projective. Consider the short exact sequence $\pi$. $\xi$ obtained by a pushout of $\xi$ along the projection $\pi: \Omega(M) \rightarrow P^{\prime}$. Note that $\operatorname{Ext}_{A}^{1}\left(M, P^{\prime}\right)=0$, since $M$ is Gorenstein projective. Then the sequence $\pi . \xi$ splits. This proves that the natural monomorphism $P^{\prime} \hookrightarrow P$ splits, which contradicts the assumption that $p$ is a projective cover. We are done.

We are now in a position to prove Theorem 1.1.

Proof of Theorem 1.1. Assume that the algebra $A$ is not CM-free. Take $M \in$ $A$-Gproj to be indecomposable and non-projective. Recall that $\mathbf{r}^{2}=0$. By Lemma 2.1 we have $\mathbf{r} M=0$, and then $M$ is semi-simple. Since $M$ is indecomposable, we conclude that $M$ is a simple module.

Set $S_{1}=M$ to be the above simple module. Take a short exact sequence $\eta: 0 \rightarrow S_{2} \stackrel{i_{2}}{\rightarrow} P_{1} \stackrel{\pi_{1}}{\rightarrow} S_{1} \rightarrow 0$ such that $\pi_{1}$ is a projective cover. By Lemma 2.2 the module $S_{2}$ is Gorenstein projective, which is indecomposable and non-projective; in particular, it is non-zero. Then by the above we infer that $S_{2}$ is a simple module. Moreover, we claim that a simple $A$-module $S$ with $\operatorname{Ext}_{A}^{1}\left(S, S_{2}\right) \neq 0$ is isomorphic to $S_{1}$.

To prove the claim, let us assume on the contrary that $S$ is not isomorphic to $S_{1}$. Take a short exact sequence $0 \rightarrow K \rightarrow P \stackrel{\pi}{\rightarrow} S \rightarrow 0$ such that $\pi$ is a projective cover. Note that the module $K$ is semi-simple, since $K \subseteq \mathbf{r} P$ and then $\mathbf{r} K=0$. Observe that $\operatorname{Ext}_{A}^{1}\left(S, S_{2}\right) \neq 0$ implies that $\operatorname{Hom}_{A}\left(K, S_{2}\right) \neq 0$. Then $S_{2}$ is a direct summand of $K$. Thus we get a non-zero morphism $S_{2} \hookrightarrow K \hookrightarrow P$ which is denoted by $l$. Note that $\operatorname{Ext}_{A}^{1}\left(S_{1}, P\right)=0$, since the module $S_{1}$ is Gorenstein projective. By the long exact sequence obtained by applying $\operatorname{Hom}_{A}(-, P)$ to $\eta$ we have an epimorphism $\operatorname{Hom}_{A}\left(P_{1}, P\right) \rightarrow \operatorname{Hom}_{A}\left(S_{2}, P\right)$ induced by $i_{2}$. Then it follows that there exists a morphism $a: P_{1} \rightarrow P$ such that $a \circ i_{2}=l$. Observe that $S_{2}$ is the socle of $P_{1}$ on which $a$ is non-zero. It follows that the morphism $a$ is mono. On the other hand, since $S$ is not isomorphic to $S_{1}$, the composite $P_{1} \stackrel{a}{\rightarrow} P \stackrel{\pi}{\rightarrow} S$ is necessarily zero. This implies that the monomorphism $a$ factors through $K$. Observe that $K$ is semi-simple, while the module $P_{1}$ is not semi-simple. This is absurd. We are done with the claim.

Similarly we define $S_{3}$ by the short exact sequence $0 \rightarrow S_{3} \stackrel{i_{3}}{\rightarrow} P_{2} \stackrel{\pi_{2}}{\rightarrow} S_{2} \rightarrow 0$ such that $\pi_{2}$ is a projective cover. As above the module $S_{3}$ is simple and non-projective, which is Gorenstein projective and satisfies the fact that any simple $A$-module $S$ with $\operatorname{Ext}_{A}^{1}\left(S, S_{3}\right) \neq 0$ is isomorphic to $S_{2}$. In this way we define $S_{n}$ for each $n \geq 1$.

Choose $n \geq 1$ minimal with the property that $S_{n} \simeq S_{m}$ for some $m<n$. Then such an $m$ is unique. We observe that $m=1$. Otherwise, we have $\operatorname{Ext}_{A}^{1}\left(S_{m-1}, S_{n}\right) \simeq$ $\operatorname{Ext}_{A}^{1}\left(S_{m-1}, S_{m}\right) \neq 0$, while $S_{m-1}$ is not isomorphic to $S_{n-1}$ by the minimality of $n$. This contradicts the claim above for $S_{n}$.

We now get a set $\left\{S_{1}, S_{2}, \cdots, S_{n-1}\right\}$ of pairwise non-isomorphic simple $A$-modules; moreover, each $S_{i}$ satisfies that any simple $A$-module $S$ with $\operatorname{Ext}_{A}^{1}\left(S, S_{i}\right) \neq 0$ is isomorphic to $S_{i-1}$. Observe that $S_{i+1} \simeq \Omega\left(S_{i}\right)$. Then we have that any simple $A$-module $S$ with $\operatorname{Ext}_{A}^{1}\left(S_{i}, S\right) \neq 0$ is isomorphic to $S_{i+1}$. Here we identify $S_{0}$ with $S_{n-1}$ and identify $S_{n}$ with $S_{1}$. Consider the valued quiver $Q_{A}$ of $A$. It then follows that the full subquiver of $Q_{A}$ with vertices $\left\{S_{1}, S_{2}, \cdots, S_{n-1}\right\}$ is a connected component. Since the algebra $A$ is connected, these are all the simple 
$A$-modules. Then accordingly all the indecomposable projective $A$-modules are given by $\left\{P_{1}, P_{2}, \cdots, P_{n-1}\right\}$. Observe from the construction of $S_{i}$ 's that each of the $P_{i}$ 's has length two and has a pairwise non-isomorphic simple socle. It follows immediately from [11, Theorem 9.3.7] that the algebra $A$ is self-injective.

Proof of Corollary 1.3. We recall some standard facts on artin algebras. Let $A$ be an artin algebra with radical square zero. Consider its valued quiver $Q_{A}$. Then $A$ has finite global dimension if and only if $Q_{A}$ does not have oriented cycles. The "if" part follows from a general result ([11, Chapter 11, Ex. 12(i)]). For the "only if" part, assume that $A$ has finite global dimension. We observe that for two simple modules $S, S^{\prime}$ with $\operatorname{Ext}_{A}^{1}\left(S, S^{\prime}\right) \neq 0, S^{\prime}$ is a direct summand of $\Omega(S)$. Here we use the fact that $\mathbf{r}^{2}=0$, and then $\Omega(S)$ is semi-simple. It then follows that proj.dim $S^{\prime} \leq$ proj.dim $S-1$, where proj.dim $X$ denotes the projective dimension of a module $X$. This inequality forces the fact that $Q_{A}$ has no oriented cycles.

Let $A$ be a connected artin algebra with radical square zero. We also need the following fact: the algebra $A$ is self-injective if and only if $Q_{A}$ is an oriented cycle with the trivial valuation. For the "only if" part, we assume that $A$ is self-injective. By [2, Proposition IV.2.16] the algebra $A$ is serial. Then the result follows from [11, Theorem 10.4.1 and Corollary 10.4.2]. For the "only if" part, we observe that all the indecomposable projective modules of the algebra A have length two and that their socles are pairwise non-isomorphic. Then it follows from 11, Theorem 9.3.7] that $A$ is self-injective.

We apply the above-recalled two facts. Then the result follows from Corollary 1.2 immediately.

\section{ACKNOWLEDGEMENTS}

This research was carried out during the author's visit to the University of Paderborn with support from Alexander von Humboldt Stiftung. He would like to thank Professor Henning Krause and the faculty of the Institut für Mathematik for their hospitality. The author is grateful to the referee for helpful suggestions. He also would like to thank Professor Zhaoyong Huang and Dr. Nan Gao for their interesting comments.

\section{REFERENCES}

1. M. Auslander and M. Bridger, Stable module category, Mem. Amer. Math. Soc. 94, 1969. MR 0269685(42:4580)

2. M. Auslander, I. Reiten, and S.O. Smalø, Representation Theory of Artin Algebras, Cambridge Studies in Adv. Math. 36, Cambridge Univ. Press, Cambridge, 1995. MR1314422 (96c:16015)

3. L.L. Avramov and A. Martsinkovsky, Absolute, relative and Tate cohomology of modules of finite Gorenstein dimension, Proc. London Math. Soc. (3) 85 (2002), 393-440. MR1912056 (2003g:16009)

4. A. Beligiannis, Cohen-Macaulay modules, (co)torsion pairs and virtually Gorenstein algebras, J. Algebra 288 (2005), 137-211. MR2138374 (2006i:16017)

5. A. Beligiannis, On rings and algebras of finite Cohen-Macaulay type, Adv. Math. 226 (2) (2011), 1973-2019.

6. R.O. Buchweitz, Maximal Cohen-Macaulay Modules and Tate Cohomology over Gorenstein Rings, unpublished manuscript, 1987.

7. X.W. Chen, An Auslander-type result for Gorenstein-projective modules, Adv. Math. 218 (2008), 2043-2050. MR2431669 (2009c:16037)

8. X.W. Chen, Singularity categories, Schur functors and triangular matrix rings, Algebr. Represent. Theor. 12 (2009), 181-191. MR2501179 (2010c:18015) 
9. X.W. Chen, Relative singularity categories and Gorenstein-projective modules, Math. Nathr. 284 (No. 2-3) (2011), 199-212.

10. L.W. Christensen, G. Piepmeyer, J. Striuli and R. Takahashi, Finite Gorenstein representation type implies simply singularity, Adv. Math. 218 (2008), 1012-1026. MR.2419377 (2009b:13058)

11. Yu A. Drozd and V.V. Kirichenko, Finite Dimensional Algebras, Springer-Verlag, Berlin, Heidelberg, 1994. MR1284468 (95i:16001)

12. E. Enochs and O. Jenda, Gorenstein injective and projective modules, Math. Z. 220 (1995), 611-633. MR1363858 (97c:16011)

13. D. Happel, Triangulated Categories in the Representation Theory of Finite Dimensional Algebras, London Math. Soc., Lecture Notes Ser. 119, Cambridge Univ. Press, Cambridge, 1988. MR935124 (89e:16035)

14. D. Happel, On Gorenstein algebras, in: Progress in Math. 95, Birkhäuser Verlag, Basel, 1991, 389-404. MR1112170 (92k:16022)

15. R. Luo and Z.Y. Huang, When are torsionless modules projective? J. Algebra 320 (2008), 2156-2164. MR2437647 (2009i:16009)

16. N. Mahdou and K. Ouarghi, Rings over which all (finitely generated strongly) Gorenstein projective modules are projective, arXiv:0902.2237v3.

17. M.S. Menzin, The condition $\operatorname{Ext}^{i}(M, R)=0$ for modules over local artin algebras $(R, \Re)$ with $\mathfrak{R}^{2}=0$, Proc. Amer. Math. Soc. 43 (1) (1974), 47-52. MR0330227 (48:8565)

18. D. Quillen, Higher algebraical K-theory I, Springer Lecture Notes in Math. 341, 1973, 85-147. MR0338129 (49:2895)

19. Y. Yoshino, Modules of G-dimension zero over local rings with the cube of maximal ideal being zero, in: Commutative algebra, singularities and computer algebra (Sinaia, 2002), NATO Sci. Ser. II Math. Phys. Chem. 115, Kluwer, Dordrecht, 2003, 255-273. MR2030276 (2004m:13039)

Wu Wen-Tsun Key Laboratory of Mathematics, University of Science and Technology of China, Chinese Academy of Sciences, Hefei 230026, Anhui, People's Republic of CHINA

E-mail address: xwchen@mail.ustc.edu.cn 